\title{
Future challenges for health economics and health technology assessment of biological drugs
}

\author{
László Gulácsi
}

Published online: 18 April 2010

(C) Springer-Verlag 2010

In this paper, some present and future challenges of health economics and health technology assessment (HTA) of biological drugs are discussed in general with particular emphasis of their utilization in rheumatology. The development and challenges of HTA in the new European Union member states are briefly touched to evaluate how their HTA agencies are coping with the increased demand of the funders and policy makers. The most important question is whether more methodological development is needed or more local data should be required (and what kind) or more international collaboration is the solution or do we need both at the same time?

Biological agents produced through genetic engineering (the so-called biologic Disease-Modifying Antirheumatic Drugs, DMARDs; i.e. tumour necrosis-alpha inhibitors, T-cell and B-cell targeted therapies, interleukin-1, -6 inhibitors) brought significant therapeutic advantages in rheumatoid arthritis (RA) treatment by improving outcomes beyond what was possible with conventional DMARDs. Therapeutic advantages are associated with increased risk of side effects as well as significant budget impact thus high attention is paid to their clinical efficacy, effectiveness and cost-effectiveness by professionals, policy makers and funders. This increasing interest can be clearly mapped in the medical literature: in PubMed, the number of publications indexed to medical subheadings "Arthritis, Rheumatoid" and ("Cost and Cost Analysis" or "Economics"

L. Gulácsi ( $\square)$

Health Economics and Health Technology Assessment Research Centre, Corvinus University of Budapest, Fovam ter 8,

1093 Budapest, Hungary

e-mail: laszlo.gulacsi@uni-corvinus.hu

URL: hecon.uni-corvinus.hu or "Cost of Illness") has increased from 261 to 873 since the registration of the first biological drug in RA (19982010). However, one decade after the introduction of the first biological agent, we are facing new challenges and some "old ones" are still unanswered. The most important ones are as follows:

\section{Changing design of RCTs}

Comparing previous and latest biological RCTs in RA, the most important differences are related to the discontinuation rate in the placebo arm. This rate was around $20-25 \%$ in the first trials whilst $80 \%$ in recently published RCTs. The clue is in the study design. Patients were followed for 24 weeks both on placebo and on active arm in the first RCTs as there were no available alternative active treatment. Nowadays, the duration of placebo control must be limited (3-6 months), and study protocols predefine withdrawal from placebo weeks/months before the primary end point is evaluated and allow patients to enter into an open label extension study. Losing the majority of the patients in the placebo arm makes the evaluation of efficacy and safety of the study drug rather difficult, and it is even more challenging to perform indirect comparisons, especially meta-analysis with previously registered comparators.

\section{Compliance, adherence and persistence}

Modelling has become a standard methodology of economic evaluation in RA offering estimates on cost-effectiveness based on RCT data for decision-makers soon after drug registration. However, the degree to which a patient correctly follows medical advice and adheres to a 
therapeutic regimen (including suboptimal compliance and/or decreased persistence) often differs between RCTs and real-life practice in all therapeutic areas. Variations in patient medication adherence may reduce the therapeutic potential of drug treatments altering its cost-effectiveness as well. A literature review recently conducted by Harrold and Andrade [5] found eleven studies evaluating adherence to pharmacologic treatments for chronic rheumatic conditions including RA. However, most of the reviewed studies looked at adherence with NSAIDs and traditional DMARDs and generally had low sample sizes. Similar to other fields of medicine, the review concluded that there was inconsistency in definitions and measurement of adherence used. Therefore, different patient populations are evaluated with different methods, which make it difficult to draw conclusions from these studies. Although more and more registries are maintained in this field providing information about effectiveness and persistence (discontinuations, switching and treatment chains) only few studies deal with compliance to biologicals in RA. Suboptimal compliance, especially with self-injectable biologicals can easily remain hidden leading to unnecessary change in treatment and further health care utilization. Some cost-effectiveness analysis based on real-world patient-level data has been published recently (e.g. Sweden, Italy) including discontinuation rates with biologicals from current clinical practice. Nevertheless, incorporating compliance in RA costeffectiveness models is still unsolved due to scarcity of appropriate input data.

\section{Epidemiology transition from prevalence-based to incidence-based approach}

Due to the lack of registries as well as shortage of systematic data collection and analysis in several European countries especially in Eastern Europe, it is difficult to estimate the size of the target population [8]. For instance, a decade ago in Hungary, the prevalence of RA was estimated based on the international literature revealing a prevalence rate of $1.0-1.5 \%$ i.e. $100,000-150,000$ patients. According to latest studies conducted in this field, the RA prevalence is about $0.37-0.5 \%$, i.e. around $50,000 \mathrm{RA}$ patients [4]. However, not more than 20 thousand RA patients visit a specialist regularly. These uncertainties have significant impact on the calculation of the size of the target population, which is a keypoint especially if the proposed therapy is costly and having potentially significant budget impact. Even less is known regarding the incidence of RA. More and more clinical trials focus on early treatment, and there is a tendency of involving patients into active care in earlier disease stage. Therefore, target population estimation is changing from prevalencebased to incidence-based approach.

\section{Do we have enough research results to be transferred among countries?}

A large number of randomized clinical trials (RCT) are available in the field of RA and other diseases such as ankylosing spondylitis (AS), psoriatic arthritis (PsA) and juvenile idiopathic arthritis (JIA). Literature on disease burden is sufficient in RA (Belgium, Denmark, Hungary, Germany, France, Italy, Spain, Sweden, the Netherlands and United Kingdom) somewhat less extent in AS (Belgium, France, Germany, The Netherlands, Spain, United Kingdom), but very limited in PsA (Germany, Italy and Hungary) and JIA (Germany, UK) [7, 8]. To transfer research results from other jurisdictions to our setting is a difficult task, even in a well researched and published medical field. Having just one or two studies in a given topic including selected patient population is even more difficult to transfer.

\section{Challenges of tomorrow}

The number of biological agents is rapidly growing in various medical fields, such as rheumatoid arthritis (early treatment), osteoporosis, mental health, multiple sclerosis and several orphan diseases. The number of new indications of the already registered and utilized biological agents is growing creating new challenges of HTA agencies to cope with. In the coming years, a growing number of pharmacoeconomic dossiers will be submitted to the funding agencies in all European countries, and decisions will have to be made somehow based on the existing (scarce) information at the point of the reimbursement decision. In the first stage of the market life of the new biological agents, these information will be almost exclusively from RCTs. However, real-life outcomes are different from those of RCTs, but from where the necessary effectiveness data will come from and when?

New member states; capacity to cope with this challenge

Cost-effectiveness is among the key information sources for health policy and financing, and it is time for a common standard for cost-effectiveness in the new member states similarly to countries with developed health economics and HTA $[3,6]$. However, 20 years after the political changes, there are only two countries in the new member states with formal HTA: Hungary and Poland. This low number 
indicates that the difficulties are underestimated or partly misunderstood in the process of the HTA development. More than 10 years history of HTA in Hungary and Poland, the very slowly changing behaviour of physicians, politicians, funders emphasizes the importance of the behavioural factors of the HTA development. We have to concentrate on that aspects as well, in order to make a change.

Other important HTA-related policy issues should have more attention as well:

In these countries, HTA is not informing health policy but is used as a tool to evaluate the economic dossiers submitted by the companies. HTA has not been recognized as a sophisticated and powerful tool for informing policy makers yet. Policy making, public health, curative medicine, management and funding are separated activities from each others, and HTA has not been yet integrated. In this present scheme, HTA is somewhat used for informing reimbursement decisions. This can be considered as a big achievement compared to the situation 5 years ago when health economics was not considered at all for funding decisions. But in order to utilize the full power of HTA, it should be integrated in policy making, public health, the development of the professional guidelines and management.

- The size of the target population and budget impact are often unknown. According to a small scale survey in Hungary, even in more than $60 \%$ of the company submitted economic dossiers estimation of the size and the budget impact for Hungary was not available.

- Very limited HTA capacity, without prioritizing HTA. In the new member states probably not more than two dozens of trained, PhD level professionals are working in this field, in some countries no professionals at all. What makes things even more difficult, no HTA topic selection process has been implemented yet in these countries. In Hungary for instance, the governmental HTA agency (Office of Health Technology Assessment, The National Institute for Strategic Health Research of the Ministry of Health) 1st May 2004-31th December 2009 evaluated 910 company submissions.

- Transparency has not been established yet. The decision-making process is not transparent, company submissions, governmental HTA agency evaluation results and financing decisions are not available for the public, at least not in Hungary. The problem is not only ethical such as no information about how taxpayer money is spent, but more importantly (for the researchers at least) that new HTAs are not informed by the already accepted or rejected cases.

- Routine data on outcomes are neither collected nor required. The usefulness of registries is limited due to varying completeness and limited access to these data [1]. Longitudinal follow-up studies are rarely conducted, and lack of research funding is also an important barrier to obtain more information on the treatment effect. Existing data from the providers, insurance fund, and national statistics are not freely available for researchers, one must pay for them. Bureaucracy and data protection legislation is also an important barrier. It is impossible to obtain permissions to link participants' characteristics with their morbidity and health care usage data in national health insurance schemes. In Hungary, even established cohort studies cannot access mortality registers to link participants' characteristics with the national mortality register [2]. Current Hungarian legislation does not allow making a crosslink between different data sources. Therefore, data on incidence, disease progression or even survival cannot be provided, as the existing registries cannot be linked with the population registry of the Central Office for Administrative and Electronic Public Services.

\section{Conclusion}

The demand for more study results of health economics and HTA will increase for health policy making and financing decisions in the coming years. More and diverse data will be needed as we discussed earlier, and the time frame to providing this information is getting shorter. We do need more methodological development oriented research, more local data from a number of well designed, preferably longitudinal epidemiological studies in different countries and more international collaboration at the same time.

The role of international collaboration will be increasing among countries with established HTA. The capacity to benefit of the new member states from this increasing international collaboration is depending on their ability and willingness to achieve significant development to eliminate the barriers listed earlier. These countries have recently a good chance to achieve significant improvement in these fields having a fair amount of EU funds to be invested in the coming years. Lots of investment are made nowadays into health care sector (among others) in these countries such as hospitals, CTs, MRI, genetic laboratories are being built, and traditional ones are restored but according to my best knowledge, only small portion of the EU funds is spent on HTA capacity building, data collection, quality indicators, research, education and decision-making in health care.

Hope this will be changed. 


\section{References}

1. Bobak M.: CEE countries; Data availability and methodological issues. Eurohealth 15(3):14-16

2. Boncz, I., Brodszky, V., Péntek, M., Ágoston, I., Nagy, Z.S., Kárpáti, K., Kriszbacher, K., Fuszek, P., Gulácsi, L.: The disease burden of colorectal cancer in Hungary. Eur. J. Health Econ. 10(1), S35-S40 (2010)

3. Gulácsi, L., Boncz, I., Drummond, M.: Issues for countries considering introducing the fourth hurdle: the case of Hungary. Int. J. Technol. Assess. Health Care 20(2), 1-5 (2004)

4. Gulácsi, L., Brodszky, V., Péntek, M., Varga, S., Vas, G., Boncz, I.: History of health technology assessment in Hungary. Int. J. Technol. Assess Health Care. Jul;25 Suppl 1:120-126 (2009)
5. Harrold, L.R., Andrade, S.E.: Medication adherence of patients with selected rheumatic conditions: a systematic review of the literature. Semin. Arthritis Rheum. 38(5), 396-402 (2009)

6. Jönsson, B.: Time for a common standard for cost-effectiveness. Eur. J. Health Econ. 7, 223-224 (2006)

7. Kobelt, G., Jönsson, B.: The burden of rheumatoid arthritis and access to treatment: outcome and cost-utility of treatments. Eur. J. Health Econ. 8(Suppl 2), 95-106 (2008)

8. Lundkvist, J., Kastang, F., Kobelt, G.: The burden of rheumatoid arthritis and access to treatment: health burden and costs. Eur. J. Health Econ. 8(Suppl 2), S49-60 (2008) 